EPJ Web of Conferences 108, 01007 (2016)

DOI: $10.1051 /$ epjconf/201610801007

(C) Owned by the authors, published by EDP Sciences, 2016

\title{
Combinatorial Approach to Modeling Quantum Systems
}

\author{
Vladimir V. Kornyak ${ }^{1, a}$ \\ ${ }^{1}$ Laboratory of Information Technologies, Joint Institute for Nuclear Research, \\ 141980 Dubna, Moscow Region, Russia
}

\begin{abstract}
Using the fact that any linear representation of a group can be embedded into permutations, we propose a constructive description of quantum behavior that provides, in particular, a natural explanation of the appearance of complex numbers and unitarity in the formalism of the quantum mechanics. In our approach, the quantum behavior can be explained by the fundamental impossibility to trace the identity of the indistinguishable objects in their evolution. Any observation only provides information about the invariant relations between such objects.

The trajectory of a quantum system is a sequence of unitary evolutions interspersed with observations - non-unitary projections. We suggest a scheme to construct combinatorial models of quantum evolution. The principle of selection of the most likely trajectories in such models via the large numbers approximation leads in the continuum limit to the principle of least action with the appropriate Lagrangians and deterministic evolution equations.
\end{abstract}

\section{Introduction}

Any continuous physical model is empirically equivalent to a certain finite model. This is widely used in practice: solutions of differential equations by the finite difference method or by using truncated series are typical examples. It is often believed that continuous models are "more fundamental" than discrete or finite models. However, there are many indications that the nature is fundamentally discrete at small (Planck) scales, and is possibly finite. ${ }^{1}$ Moreover, the description of the physical systems by, e.g., differential equations can not be fundamental in principle, since it is based on approximations of the form $f(x) \approx f\left(x_{0}\right)+\nabla f\left(x_{0}\right) \Delta x$. In this paper we consider some approaches to constructing discrete combinatorial models of the quantum evolution.

The classical description of a reversible dynamical system looks schematically as follows. There are a set $W$ of states ${ }^{2}$ and a group $G_{\mathrm{cl}} \leq \operatorname{Sym}(W)$ of transformations (bijections) of $W$. Evolutions of $W$ are described by sequences of group elements $g_{t} \in G_{\mathrm{cl}}$ parameterized by the continuous time $t \in \mathbf{T}=\left[t_{a}, t_{b}\right] \subseteq \mathbb{R}$. The observables are functions $h: W \rightarrow \mathbb{R}$.

An arbitrary set $W$ can be "quantized" by assigning numbers from a number system $\mathcal{F}$ to the elements $w \in W$, i.e., by interpreting $W$ as a basis of the module $\mathcal{F}^{\otimes W}$. The quantum description of a

\footnotetext{
ae-mail: kornyak@jinr.ru

${ }^{1}$ The total number of binary degrees of freedom in the Universe is about $10^{122}$ as estimated via the holographic principle and the Bekenstein-Hawking formula.

${ }^{2}$ The set $W$ often has the structure of a set of functions: $W=\Sigma^{X}$, where $X$ is a space, and $\Sigma$ is a set of local states.
}

This is an Open Access article distributed under the terms of the Creative Commons Attribution License 4.0, which permits unrestricted use, distribution, and reproduction in any medium, provided the original work is properly cited. Article available at http://www.epl-conterences.org or http://dx.dol.org/10.1051/ep|cont/201610801007 
dynamical system assumes that the module spanned by the set of classical states $W$ is a Hilbert space $\mathcal{H}_{W}$ over the field of complex numbers, i.e., $\mathcal{F}=\mathbb{C}$. The transformations $g_{t}$ and the observables $h$ are replaced by unitary, $U_{t} \in \operatorname{Aut}\left(\mathcal{H}_{W}\right)$, and Hermitian, $H$, operators on $\mathcal{H}_{W}$, respectively. A constructive version of the quantum description is reduced to the following:

- time is discrete and can be represented as a sequence of integers, typically $\mathbf{T}=[0,1, \ldots, T]$;

- the set $W$ is finite and, respectively, the space $\mathcal{H}_{W}$ is finite-dimensional;

- the general unitary group Aut $\left(\mathcal{H}_{W}\right)$ is replaced by a finite group $G$;

- the field $\mathbb{C}$ is replaced by $\mathcal{K}$ th cyclotomic field $\mathbb{Q}_{\mathcal{K}}$, where $\mathcal{K}$ depends on the structure of $G$;

- the evolution operators $U_{t}$ belong to a unitary representation of $G$ in the Hilbert space $\mathcal{H}_{W}$ over $\mathbb{Q}_{\mathcal{K}}$.

It is clear that a single unitary evolution is not sufficient for describing the physical reality. Such evolution is nothing more than a physically trivial change of coordinates (a symmetry transformation). This means that observable values or relations, being invariant functions of states, do not change with time. As an example, consider a unitary evolution of a pair of state vectors: $\left|\varphi_{1}\right\rangle=U\left|\varphi_{0}\right\rangle$, $\left|\psi_{1}\right\rangle=U\left|\psi_{0}\right\rangle$. For the scalar product we have $\left\langle\varphi_{1} \mid \psi_{1}\right\rangle=\left\langle\varphi_{0}\left|U^{-1} U\right| \psi_{0}\right\rangle \equiv\left\langle\varphi_{0} \mid \psi_{0}\right\rangle$. There are two ways to obtain observable effects in the scenario of unitary evolution: (a) in quantum mechanics measurements are described by non-unitary operators - projections into subspaces of the Hilbert space; (b) in gauge theories collections of evolutions are considered, and comparing results of different evolutions can lead to observable effects (in the case of a non-trivial gauge holonomy).

The role of observations in quantum mechanics is very important - it is sometimes said that "observation creates reality". ${ }^{3}$ We pay special attention to the explicit inclusion of observations in the models of evolution. While the states of a system are fixed in the moments of observation, there is no objective way to trace the identity of the states between observations. In fact, all identifications i.e., parallel transports provided by the gauge group which describes symmetries of the states - are possible. This leads to a kind of fundamental indeterminism. To handle this indeterminism we need a way to describe statistically collections of parallel transports. Then we can formulate the problem of finding trajectories with maximum probability that pass through a given sequence of states fixed by observations. In a properly formulated model, the principle of selection of the most probable trajectories should reproduce in the continuum limit the principle of the least action.

\section{Constructive description of quantum behavior}

The transition from a continuous quantum problem to its constructive counterpart can be done by replacing a unitary group of evolution operators with some finite group. To justify such a replacement [1] one can use the fact from the theory of quantum computing that any unitary group contains a dense finitely generated subgroup. This residually finite [2] group has infinitely many finite homomorphic images. The infinite set of non-trivial homomorphisms allows to find a finite group that is empirically equivalent to the original unitary group in any particular problem.

\subsection{Permutations and natural quantum amplitudes}

As it is well known, any representation of a finite group is a subrepresentation of some permutation representation. Namely, a representation $U$ of $G$ in a K-dimensional Hilbert space $\mathcal{H}_{\mathrm{K}}$ can be embedded into a permutation representation $\mathrm{P}$ of $\mathrm{G}$ in an $\mathrm{N}$-dimensional Hilbert space $\mathcal{H}_{\mathrm{N}}$, where

\footnotetext{
${ }^{3}$ The phrase is often attributed to John Archibald Wheeler.
} 
$N \geq K$. The representation $P$ is equivalent to an action of $G$ on a set of things $\Omega=\left\{\omega_{1}, \ldots, \omega_{N}\right\}$ by permutations. If $\mathrm{K}=\mathrm{N}$ then $\mathrm{U} \cong \mathrm{P}$. Otherwise, if $\mathrm{K}<\mathrm{N}$, the embedding has the structure

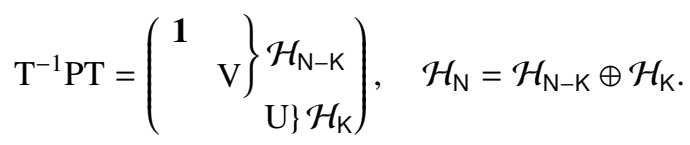

Here 1 is the trivial one-dimensional representation. It is a mandatory subrepresentation of any permutation representation. $\mathrm{V}$ is an optional subrepresentation. We can treat the unitary evolutions of data in the spaces $\mathcal{H}_{\mathrm{K}}$ and $\mathcal{H}_{\mathrm{N}-\mathrm{K}}$ independently, since both spaces are invariant subspaces of $\mathcal{H}_{\mathrm{N}}$.

The embedding into permutations provides a simple explanation of the presence of complex numbers and complex amplitudes in the formalism of the quantum mechanics. We interpret complex quantum amplitudes as projections onto invariant subspaces of vectors with natural components for a suitable permutation representation $[1,3,4]$. It is natural to assign natural numbers - multiplicities — to elements of the set $\Omega$ on which the group $G$ acts by permutations. The vector of multiplicities,

$$
|n\rangle=\left(\begin{array}{c}
n_{1} \\
\vdots \\
n_{\mathrm{N}}
\end{array}\right)
$$

is an element of the module $\mathrm{H}_{N}=\mathbb{N}^{N}$, where $\mathbb{N}=\{0,1,2, \ldots\}$ is the semiring of natural numbers. The permutation action defines the permutation representation of $\mathrm{G}$ in the module $\mathrm{H}_{\mathrm{N}}$. Using the fact that all eigenvalues of any linear representation of a finite group are roots of unity, we can turn the module $\mathrm{H}_{\mathrm{N}}$ into a Hilbert space $\mathcal{H}_{\mathrm{N}}$. We denote by $\mathbb{N}_{\mathcal{K}}$ the semiring formed by linear combinations of $\mathcal{K}$ th roots of unity with natural coefficients. The so-called conductor $\mathcal{K}$ is a divisor of the exponent ${ }^{4}$ of $\mathrm{G}$. In the case $\mathcal{K}>1$ the semiring $\mathbb{N}_{\mathcal{K}}$ becomes a ring of cyclotomic integers. The introduction of the cyclotomic field $\mathbb{Q}_{\mathcal{K}}$ as the field of fractions of the ring $\mathbb{N}_{\mathcal{K}}$ completes the conversion of the module $\mathrm{H}_{\mathrm{N}}$ into the Hilbert space $\mathcal{H}_{\mathrm{N}}$. If $\mathcal{K}>2$, then $\mathbb{Q}_{\mathcal{K}}$ is empirically equivalent to the field of complex numbers $\mathbb{C}$ in the sense that $\mathbb{Q}_{\mathcal{K}}$ is a dense subfield of $\mathbb{C}$.

\subsection{Measurements and the Born rule}

A quantum measurement is, in fact, a selection among all the possible state vectors that belong to a given subspace of a Hilbert space. This subspace is specified by the experimental setup. The probability to find a state vector in the subspace is described by the Born rule. There have been many attempts to derive the Born rule from other physical assumptions — the Schrödinger equation, Bohmian mechanics, many-worlds interpretation, etc. However, the Gleason theorem [5] shows that the Born rule is a logical consequence of the very definition of a Hilbert space and has nothing to do with the laws of evolution of the physical systems.

The Born rule expresses the probability to register a particle described by the amplitude $|\psi\rangle$ by an apparatus configured to select the amplitude $|\phi\rangle$ by the formula (in the case of pure states):

$$
\mathbf{P}(\phi, \psi)=\frac{|\langle\phi \mid \psi\rangle|^{2}}{\langle\phi \mid \phi\rangle\langle\psi \mid \psi\rangle} \equiv \frac{\left\langle\psi\left|\Pi_{\phi}\right| \psi\right\rangle}{\langle\psi \mid \psi\rangle} \equiv \operatorname{tr}\left(\Pi_{\phi} \Pi_{\psi}\right),
$$

where $\Pi_{a}=\frac{|a\rangle\langle a|}{\langle a \mid a\rangle}$ is the projector onto the subspace spanned by $|a\rangle$.

Remark. In the "finite" background the only reasonable interpretation of probability is the frequency

\footnotetext{
${ }^{4}$ The exponent of a group is defined as the least common multiple of the orders of its elements.
} 
interpretation: probability is the ratio of the number of "favorable" combinations to the total number of combinations. So we expect that $\mathbf{P}(\phi, \psi)$ must be a rational number if everything is arranged correctly. Thus, in our approach the usual non-constructive contraposition - complex numbers as intermediate values vs. real numbers as observable values - is replaced by the constructive one irrationalities vs. rationals. From the constructive point of view, there is no fundamental difference between irrationalities and constructive complex numbers: both are elements of algebraic extensions.

\subsection{Illustration: constructive view of the Mach-Zehnder interferometer}

The Mach-Zehnder interferometer is a simple but important example of a two-level quantum system. The device consists of a single-photon light source, beam splitters, mirrors and photon detectors (see Figure 1). Consider a two-dimensional Hilbert space spanned by the two orthonormal basis vectors

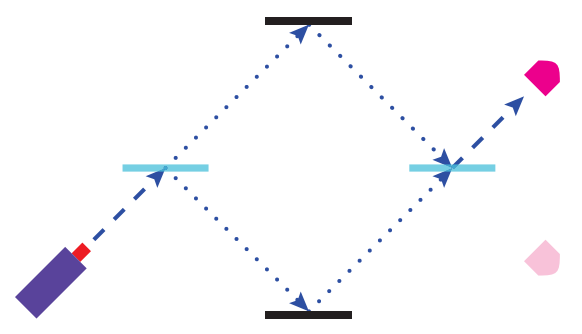

Figure 1. Mach-Zehnder interferometer. Balanced setup: both beam splitters are 50/50 and there is no phase shift between upper and lower paths.

$|\nearrow\rangle$ - "right upward beams", and $\backslash \searrow\rangle$ - "right downward beams". Then the 50/50 beam splitter (i.e., a photon has equal probability of being reflected and transmitted) is described by the matrix

$$
S=\frac{1}{\sqrt{2}}\left(\begin{array}{ll}
1 & \mathrm{i} \\
\mathrm{i} & 1
\end{array}\right) \text {. }
$$

The mirror matrix is $M=\left(\begin{array}{ll}0 & \mathrm{i} \\ \mathrm{i} & 0\end{array}\right)$. Notice that $M=S^{2}$, and, on the other hand, $S$ can be expressed via $M$ as an element of the group algebra: $S=\frac{1}{\sqrt{2}}(\mathrm{I}+M)$, where $\mathrm{I}$ is the identity matrix. The scheme in the figure implements the unitary evolution $S M S|\nearrow\rangle=S^{4}|\nearrow\rangle=-|\nearrow\rangle$, which means that only the upper detector will register photons, the lower detector will always be inactive.

This device is able to demonstrate many interesting features of the quantum behavior. Consider, for example, the scheme of quantum interaction-free measurement proposed by Elitzur and Vaidman [6]. The Penrose's version of this example is called the bomb-testing problem. Suppose we have a collection of bombs, of which some are defective. The detonator of a good bomb causes explosion after absorbing a single photon. The detonators of defective bombs reflect photons without any consequences. Classically, the only way to verify that a bomb is good is to touch the detonator. However, as shown in Figure 2, the quantum interference makes it possible to select $25 \%$ of good bombs without exploding them: the signal of the lower detector ensures that the unexploded bomb is good.

A slight modification of the scheme shown in Figure 1 allows us to implement any unitary operator $U \in \mathrm{U}(2)$ by the Mach-Zehnder interferometer. This is easily verified by direct calculation. Since $\operatorname{dim} U(2)=4$, we should add four parameters in a proper way. For example, we can change the transparency of the beam splitter. Mathematically this means replacing the matrix (1) by another one of the form $\alpha \mathrm{I}+\beta M$, where $|\alpha|^{2}+|\beta|^{2}=1$. Another possibility is to introduce phase shifters. The phase shifter matrix related, e.g., to a "right upward beam" has the form $\left(\begin{array}{cc}\mathrm{e}^{\mathrm{i} \omega} & 0 \\ 0 & 1\end{array}\right)$. Moreover, combining many Mach-Zehnder interferometers [7], one can realize elements of any unitary group $\mathrm{U}(n)$. 


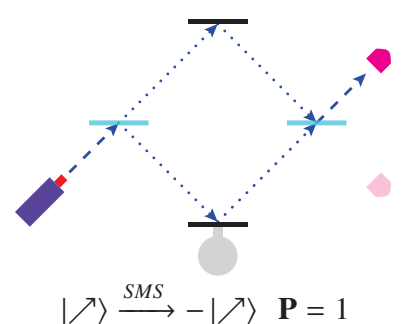

testing defective bomb

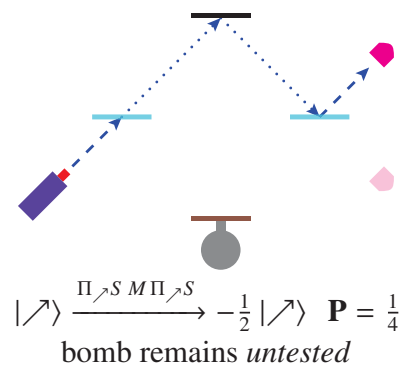

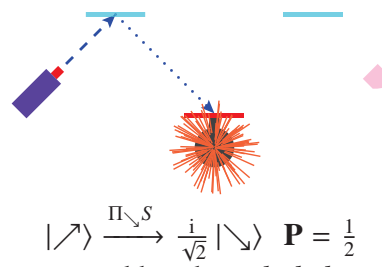

good bomb exploded

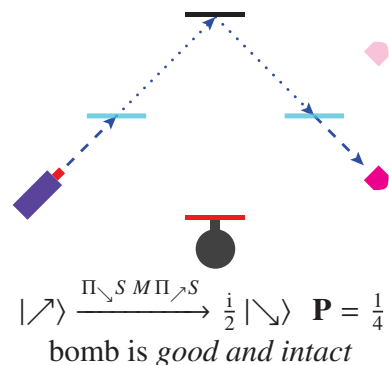

Figure 2. Penrose bomb tester. $\mathbf{P}$ is the probability of a branch of evolution. $\Pi_{a}$ denotes the projector onto $|a\rangle$.

Since a "mirror" is the square of a "beam splitter", any unitary evolution in a sequence of balanced Mach-Zehnder interferometers can be described by degrees of $S$. The operator $S$ generates the cyclic group $\mathbb{Z}_{8}$. The smallest degree faithful action of $\mathbb{Z}_{8}$ is realized by permutations of 8 objects. Any of the four permutations, that generate $\mathbb{Z}_{8}$ as a group of permutations, can be put in correspondence with the beam splitter, e.g., $S \longleftrightarrow g=(1,2,3,4,5,6,7,8)$. The generator $g$ can be represented by a matrix $P_{g}$ acting in the module $\mathbb{N}^{8}$ that consists of the vectors with natural components:

$$
N=\left(n_{1}, n_{2}, n_{3}, n_{4}, n_{5}, n_{6}, n_{7}, n_{8}\right)^{T} \in \mathbb{N}^{8} .
$$

To "extract" the beam splitter from the matrix $P_{g}$ we should extend the natural numbers by 8 th roots of unity - the conductor $\mathcal{K}=8$ in this case. Any 8 th root of unity can be represented as a power of any of the four primitive roots defined by the cyclotomic polynomial $\Phi_{8}(r)=r^{4}+1$. Let us denote by $\mathbb{N}_{8}$ the set of linear combinations of 8th roots of unity with natural coefficients. This is a ring since $\mathcal{K}=8>1$. The ring $\mathbb{N}_{8}$ is isomorphic to the ring of 8 th cyclotomic integers. In principle, due to the projective nature of the quantum states, we could perform all calculations using only natural numbers and roots of unity. But it is convenient to use also the 8th cyclotomic field, which we will denote by $\mathbb{Q}_{8}$. The field $\mathbb{Q}_{8}$ is the fraction field of the ring $\mathbb{N}_{8}$.

The matrix $P_{g}$ by a transformation $T$ over the field $\mathbb{Q}_{8}$ can be reduced to the form

$$
S_{g}=T^{-1} P_{g} T=\left(\begin{array}{cc}
A & 0 \\
0 & S_{\mathrm{r}}
\end{array}\right)
$$

where $A=\operatorname{diag}\left(1,-1, \mathrm{r}^{2},-\mathrm{r}^{2}, \mathrm{r}^{3},-\mathrm{r}\right), \mathrm{r}$ is a primitive 8 th root of unity, and

$$
S_{\mathrm{r}}=\frac{1}{2}\left(\begin{array}{ll}
\mathrm{r}-\mathrm{r}^{3} & \mathrm{r}+\mathrm{r}^{3} \\
\mathrm{r}+\mathrm{r}^{3} & \mathrm{r}-\mathrm{r}^{3}
\end{array}\right)
$$


is the beam splitter matrix $S$ expressed in terms of cyclotomic numbers. The quantum amplitude of the Mach-Zehnder interferometer can be approximated by the projection of the natural vector $N$ into the "splitter" subspace:

$$
|\psi\rangle=\left(\begin{array}{l}
\psi_{1} \\
\psi_{2}
\end{array}\right)=\frac{1}{8}\left(\begin{array}{c}
-\mathrm{r}^{3}\left(n_{1}+n_{3}-n_{5}-n_{7}\right)+\left(1-\mathrm{r}^{2}\right)\left(n_{2}-n_{6}\right) \\
\mathrm{r}\left(n_{1}-n_{3}-n_{5}+n_{7}\right)+\left(1+\mathrm{r}^{2}\right)\left(-n_{4}+n_{8}\right)
\end{array}\right) .
$$

It can be shown that the expression (3) can approximate with arbitrary precision any point on the Bloch sphere - a standard representation of the complex projective line $\mathbb{C} P^{1}$.

\section{Combinatorial models of evolution}

Let us begin with some general considerations concerning the evolution of the probabilistic systems subject to observations. The evolution of such a system can be described as follows. We have a fundamental ("Planck") time which is the sequence of integers:

$$
\mathbf{T}=[0,1, \ldots, T] .
$$

There is also a sequence of "times of observations". For simplicity, we assume that the observation time is a subsequence of the fundamental time

$$
\mathcal{T}=\left[t_{0}=0, \ldots, t_{i-1}, t_{i}, \ldots, t_{N}=T\right]
$$

(otherwise we could assume that the times of the observations are not determined exactly, e.g., they could be random variables with probability distributions localized within subintervals of the fundamental time). Let $W_{t_{i}}$ denote the state of a system observed at the time $t_{i}$, and

$$
W_{t_{0}} \rightarrow \cdots \rightarrow W_{t_{i-1}} \rightarrow W_{t_{i}} \rightarrow \cdots \rightarrow W_{t_{N}}
$$

denote a trajectory of the system. Whereas the states $W_{t_{i-1}}$ and $W_{t_{i}}$ are fixed by observation, the transition between them can be described only probabilistically.

The selection of the most probable trajectories is the main problem in the study of the evolution. If we can specify $\mathbf{P}_{W_{t_{i-1}} \rightarrow W_{t_{i}}}$ - the one-step transition probability - then the probability of trajectory (6) can be calculated as the product

$$
\mathbf{P}_{W_{t_{0}} \rightarrow \cdots \rightarrow W_{t_{N}}}=\prod_{i=1}^{N} \mathbf{P}_{W_{t_{i-1}} \rightarrow W_{t_{i}}} .
$$

The inconvenience of dealing with the product of large number of multipliers can be eliminated by introducing the entropy, which is defined as the logarithm of probability. The transition to logarithms allows us to replace the products by sums. On the other hand, taking the logarithm does not change the positions of the extrema of a function due to the monotonicity of the logarithm. Thus, for searching the most likely trajectories we introduce the one-step entropy

$$
\mathbf{S}_{W_{t_{i-1}} \rightarrow W_{t_{i}}}=\log \mathbf{P}_{W_{t_{i-1}} \rightarrow W_{t_{i}}}
$$

and use instead of (7) the entropy of trajectory:

$$
\mathbf{S}_{W_{t_{0}} \rightarrow \cdots \rightarrow W_{t_{N}}}=\sum_{i=1}^{N} \mathbf{S}_{W_{t_{i-1}} \rightarrow W_{t_{i}}} .
$$


The formulation of any dynamical model usually begins with postulating a Lagrangian. However, it would be desirable to derive Lagrangians from more fundamental principles. One can see that continuum approximations of (8) and (9) lead to the concepts of Lagrangian and action, respectively. The reasoning is schematically the following. The states $W_{t_{i}}$ are specified by sets of numerical parameters (coordinates) $\mathbf{X}_{t_{i}}=\left(X_{1, t_{i}}, X_{2, t_{i}}, \ldots, X_{K, t_{i}}\right)$. For a specific model one-step entropy (8) can be calculated as a function of the coordinates: $\mathbf{S}_{W_{t_{i-1}} \rightarrow W_{t_{i}}}=S\left(\mathbf{X}_{t_{i}}, \Delta \mathbf{X}_{t_{i}}\right)$, where $\Delta \mathbf{X}_{t_{i}}=\mathbf{X}_{t_{i}}-\mathbf{X}_{t_{i-1}}$. Assuming that $N \rightarrow \infty, t_{i}-t_{i-1} \rightarrow 0$ and embedding the sequence $\mathbf{X}_{t_{i}}$ into the continuous function $\mathbf{X}(t)$, we can represent the one-step entropy in the form $S\left(\mathbf{X}\left(t_{i}\right), \Delta \mathbf{X}\left(t_{i}\right)\right)$. The second order Taylor approximation of this function has the form $S \approx A+b_{k k^{\prime}}\left(\Delta X_{k}\left(t_{i}\right)-\Delta X_{k}^{*}\left(t_{i}\right)\right)\left(\Delta X_{k^{\prime}}\left(t_{i}\right)-\Delta X_{k^{\prime}}^{*}\left(t_{i}\right)\right)$, where $\Delta \mathbf{X}^{*}\left(t_{i}\right)$ is the solution of the system of equations $\frac{\partial S}{\partial \Delta \mathbf{X}\left(t_{i}\right)}=0$. Since the discrete time is a dimensionless counter, the differences can be approximated in the continuum limit by introducing derivatives, and we come to the Lagrangian

$$
\mathcal{L}=A+B_{k k^{\prime}}\left(\frac{d X_{k}}{d t}-a_{k}\right)\left(\frac{d X_{k^{\prime}}}{d t}-a_{k^{\prime}}\right),
$$

where $B_{k k^{\prime}}$ is a negative definite quadratic form; $B_{k k^{\prime}}, A$ and $a_{k}$ depend on $X_{1}(t), X_{2}(t), \ldots, X_{K}(t)$. The action

$$
\mathcal{S}=\int \mathcal{L} d t
$$

is a continuum approximation of the entropy of trajectory (9), so the principle of least action can be treated as a continuous remnant of the principle of selection of the most likely trajectories.

\subsection{Example: extracting Lagrangian from combinatorics}

As an illustration of the above let us consider the one-dimensional random walk. This model studies the statistics of sequences of positive $(+1)$ and negative $(-1)$ unit steps on the integer line $\mathbb{Z}$. Any statistical description is based on the concepts of microstates and macrostates - the last can naturally be treated as equivalence classes of microstates [8]. In this model, microstates are individual sequences of steps. The probability of a microstate consisting of $k_{+}$positive and $k_{-}$negative steps is equal to $\alpha_{+}^{k_{+}} \alpha_{-}^{k_{-}}$, where $\alpha_{+}$and $\alpha_{-}$denote probabilities of single steps $\left(\alpha_{+}+\alpha_{-}=1\right)$. The macrostates are defined by the equivalence relation: two sequences $u$ and $v$ are equivalent if $k_{+}^{u}+k_{-}^{u}=k_{+}^{v}+k_{-}^{v}=t$ and $k_{+}^{u}-k_{-}^{u}=k_{+}^{v}-k_{-}^{v}=x$, i.e., both sequences have the same length $t$ and define the same point $x$ on $\mathbb{Z}$. The probability of an arbitrary microstate to belong to a given macrostate is described by the binomial distribution, which in terms of the variables $x$ and $t$ takes the form

$$
P(x, t)=\frac{t !}{\left(\frac{t+x}{2}\right) !\left(\frac{t-x}{2}\right) !}\left(\frac{1+v}{2}\right)^{\frac{t+x}{2}}\left(\frac{1-v}{2}\right)^{\frac{t-x}{2}},
$$

where $v=\alpha_{+}-\alpha_{-}$is the "drift velocity". ${ }^{5}$ Obviously, $-1 \leq v \leq 1$.

Let $\left[x_{0}, \ldots, x_{i-1}, x_{i}, \ldots, x_{N}\right]$ be a sequence of points (observed values) corresponding to the sequence of times of observations (5). We assume that the time differences $\Delta t_{i}=t_{i}-t_{i-1}$ are much larger than the unit of fundamental time (4) but much less than the total time: $1 \ll \Delta t_{i} \ll T$. Applying formula (10) to $i$ th time interval we can write the one-step entropy:

$$
\mathbf{S}_{x_{i-1} \rightarrow x_{i}}=\ln \Delta t_{i} !-\ln \left(\frac{\Delta t_{i}+\Delta x_{i}}{2}\right) !-\ln \left(\frac{\Delta t_{i}-\Delta x_{i}}{2}\right) !+\frac{\Delta t_{i}+\Delta x_{i}}{2} \ln \left(\frac{1+v_{i}}{2}\right)+\frac{\Delta t_{i}-\Delta x_{i}}{2} \ln \left(\frac{1-v_{i}}{2}\right),
$$

\footnotetext{
${ }^{5}$ It has been shown [9] that the velocity, defined in a similar way, i.e., as the difference of probabilities of steps in opposite directions, satisfies the relativistic velocity addition rule: $w=(u+v) /(1+u v)$.
} 
where $\Delta x_{i}=x_{i}-x_{i-1}$, and $v_{i}$ denotes the drift velocity in the $i$ th interval.

Applying the Stirling approximation, $\ln n ! \approx n \ln n-n$, we have

$$
\mathbf{S}_{x_{i-1} \rightarrow x_{i}} \approx S_{i}=\Delta t_{i} \ln \Delta t_{i}-\frac{\Delta t_{i}+\Delta x_{i}}{2} \ln \left(\frac{\Delta t_{i}+\Delta x_{i}}{1+v_{i}}\right)-\frac{\Delta t_{i}-\Delta x_{i}}{2} \ln \left(\frac{\Delta t_{i}-\Delta x_{i}}{1-v_{i}}\right) .
$$

Solving the equation $\partial S_{i} / \partial \Delta x_{i}=0$ we obtain the stationary point: $\Delta x_{i}^{*}=v_{i} \Delta t_{i}$. Replacing the sequences $x_{i}, v_{i}$ by continuous functions $x(t), v(t)$ and introducing the approximation $\Delta x_{i} \approx \dot{x}(t) \Delta t_{i}$ in the second order Taylor expansion of (11) around the point $\Delta x_{i}^{*}$ we have finally

$$
\mathbf{S}_{x_{i-1} \rightarrow x_{i}} \approx-\frac{1}{2}\left(\frac{\dot{x}(t)-v}{\sqrt{1-v^{2}}}\right)^{2} \Delta t_{i}
$$

Thus we come to the Lagrangian $\mathcal{L}=\left(\frac{\dot{x}(t)-v}{\sqrt{1-v^{2}}}\right)^{2}$ with the corresponding Euler-Lagrange equation

$$
\frac{d}{d t} \frac{\partial \mathcal{L}}{\partial \dot{x}}-\frac{\partial \mathcal{L}}{\partial x}=0 \Longrightarrow \ddot{x}\left(1-v^{2}\right)+2 \dot{x} v \frac{\partial v}{\partial t}-\left(1+v^{2}\right) \frac{\partial v}{\partial t}=0 .
$$

\subsection{Scheme for constructing models of quantum evolution}

The trajectory of a quantum system is a sequence of observations with unitary evolutions between them. We propose a scheme to construct quantum models that combine unitary evolutions with observations. The scheme assumes that transitions between observations are described by bunches of properly weighted unitary parallel transports. The standard scheme of quantum mechanics with single unitary evolutions can be reproduced in our scheme by a special choice of weights. But in our scheme such unique evolutions are assumed to be obtained as statistically dominant elements of the bunches.

We use the following notations

- $\mathcal{H}$ : a Hilbert space;

- $\Pi_{\psi_{t_{0}}}, \ldots, \Pi_{\psi_{t_{i}}}, \ldots, \Pi_{\psi_{t_{N}}}$ : a sequence of observations, where $\Pi_{\psi_{t_{i}}}=\left|\psi_{t_{i}}\right\rangle\left\langle\psi_{t_{i}}\right|$ is the projector that fixes $\psi_{t_{i}} \in \mathcal{H}$ as the result of observation at the time $t_{i}$;

- $\Delta t_{i}=t_{i}-t_{i-1}$ : the length of $i$ th time interval;

- $\mathrm{G}=\left\{\mathrm{g}_{1}, \ldots, \mathrm{g}_{\mathrm{M}}\right\}$ : a finite gauge group;

- $\mathrm{U}$ : a unitary representation of $\mathrm{G}$ in the space $\mathcal{H}$;

- $\gamma=g_{1}, \ldots, g_{\Delta t_{i}}$ : a sequence of the length $\Delta t_{i}$ of elements from G;

- $\operatorname{val}(\gamma)=\prod_{j=1}^{\Delta t_{i}} g_{j} \in \mathrm{G}$ : the (group) value of the sequence $\gamma$ - the parallel transport;

- $\Gamma_{i}=\left\{\gamma_{1}, \ldots, \gamma_{k}, \ldots, \gamma_{K_{i}}\right\}$ : an (arbitrary) enumeration of the set of all sequences $\gamma$, where $\mathrm{K}_{i} \equiv\left|\Gamma_{i}\right|=\mathrm{M}^{\Delta t_{i}}$ is the total number of the sequences;

- $w_{k i}$ : a non-negative weight of $k$ th sequence (in $i$ th time interval).

With these notations we come to the scheme shown in Figure 3. The probability of transition from $\psi_{t_{i-1}}$ to $\psi_{t_{i}}$ is given by the formula

$$
\mathbf{P}_{\psi_{t_{i-1}} \rightarrow \psi_{t_{i}}}=\sum_{k=1}^{\mathrm{K}_{i}} w_{k i}\left\langle\varphi_{k i}\left|\Pi_{\psi_{t_{i}}}\right| \varphi_{k i}\right\rangle, \text { where } \varphi_{k i}=\mathrm{U}\left(\operatorname{val}\left(\gamma_{k}\right)\right) \psi_{t_{i-1}}
$$




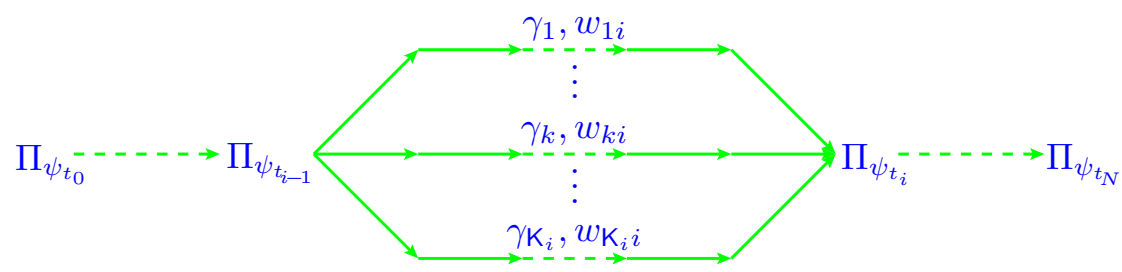

Figure 3. Scheme of quantum evolution with observations

The case of standard quantum mechanics with a single unitary evolution between observations is obtained in our scheme by selecting a sequence $\gamma$ formed by an element $g \in \mathrm{G}$ repeated $\Delta t_{i}$ times. The weight of the sequence $\gamma$ is set to 1 , and the weights of all other sequences are equated to 0 . In other words, the set of weights is the Kronecker delta on the set of sequences: $w_{k i}=\delta_{\gamma, \gamma_{k}}, \gamma_{k} \in \Gamma_{i}$. Introducing the Hamiltonian $H=\mathrm{i} \ln \mathrm{U}(g)$, we can write the evolution in the usual form

$$
U \equiv \mathrm{U}\left(g^{\Delta t_{i}}\right)=\mathrm{e}^{-\mathrm{i} H\left(t_{i}-t_{i-1}\right)} .
$$

Since the notion of Hamiltonian stems from the principle of least action, it is natural to assume the existence of some mechanism of selecting sequences of the form $g, g, \ldots, g$ as dominant elements in the set of all sequences. This requires a detailed analysis of the combinatorics of steps in fundamental time (4) for particular models.

\subsection{Dynamics of observed quantum system. Quantum Zeno effect and finite groups}

Consider the issue concerning the connection between the quantum dynamics and the group properties of unitary evolution operators. Namely, we consider the quantum Zeno effect for operators that belong to representations of finite groups.

The "quantum Zeno effect"6 (see the review [10]) is a feature of the quantum dynamics, which is manifested in the fact that frequent measurements can stop (or slow down) the evolution of a system - for example, inhibit decay of an unstable particle - or force it to evolve in a prescribed way. In the latter case, the phenomenon is called the "anti-Zeno effect".

Consider a quantum system that evolves from the initial (at $t=0$ ) normalized pure state $\left|\psi_{0}\right\rangle$ under the action of the unitary operator $U=\mathrm{e}^{-\mathrm{i} H t}$, where $H$ is the Hamiltonian. The probability to find the system in the initial state at the time $t$ is the following

$$
p_{H}(t)=\left|\left\langle\psi_{0}\left|\mathrm{e}^{-\mathrm{i} H t}\right| \psi_{0}\right\rangle\right|^{2}
$$

The most important characteristics of any dynamical process are its temporal parameters. For the quantum Zeno effect such a parameter is called the "Zeno time", denoted $\tau_{Z}$. It is determined from the short-time expansion of (12):

$$
p_{H}(t)=1-t^{2} / \tau_{Z}^{2}+\mathrm{O}\left(t^{4}\right)
$$

Calculation of (13) shows that $\tau_{Z}^{-2}=\left\langle\psi_{0}\left|H^{2}\right| \psi_{0}\right\rangle-\left\langle\psi_{0}|H| \psi_{0}\right\rangle^{2}$.

\footnotetext{
${ }^{6}$ This effect is also known under the name "the Turing paradox".
} 
Let us present the so-called Zeno dynamics in the framework of scheme proposed in Section 3.2. We have here the sequence of observations $\Pi_{\psi_{t_{0}}}, \Pi_{\psi_{t_{1}}}, \ldots, \Pi_{\psi_{t_{N}}}$, each of which selects the same state $\psi_{0}$, i.e., $\psi_{t_{0}}=\psi_{t_{1}}=\cdots=\psi_{t_{N}} \equiv \psi_{0}$. Assuming that $t_{0}=0, t_{N}=T$ and the times of observations are equidistant: $t_{i}-t_{i-1}=T / N$, we can write, using (13), the approximation for the one-step transition probability

$$
\mathbf{P}_{\psi_{t_{i-1}} \rightarrow \psi_{t_{i}}} \approx 1-\frac{1}{N^{2}}\left(\frac{T}{\tau_{Z}}\right)^{2}
$$

with the corresponding approximation for the one-step entropy

$$
\mathbf{S}_{\psi_{t_{i-1}} \rightarrow \psi_{t_{i}}} \approx-\frac{1}{N^{2}}\left(\frac{T}{\tau_{Z}}\right)^{2}
$$

For the entropy of the trajectory we have

$$
\mathbf{S}_{\psi_{t_{0}} \rightarrow \cdots \rightarrow \psi_{t_{N}}}=\sum_{i=1}^{N} \mathbf{S}_{\psi_{t_{i-1}} \rightarrow \psi_{t_{i}}} \approx-\frac{1}{N}\left(\frac{T}{\tau_{Z}}\right)^{2} \stackrel{N \rightarrow \infty}{\longrightarrow} 0
$$

and, respectively, for the probability of trajectory: $\quad \mathbf{P}_{\psi_{t_{0}} \rightarrow \cdots \rightarrow \psi_{t_{N}}} \stackrel{N \rightarrow \infty}{\longrightarrow} \mathrm{e}^{0}=1$.

This is precisely the essence of the Zeno effect.

Now assume that the evolution operator $U$ belongs to a representation of a finite group $\mathrm{G}$, i.e., $U=\mathrm{U}(\mathrm{g}), \mathrm{g} \in \mathrm{G}$, and the time is the sequence of natural numbers: $t=0,1,2, \ldots$. A natural way to define the Zeno time in this case follows from the observation that the leading part of the expansion (13) vanishes at $t=\tau_{Z}$. By analogy we can define the natural Zeno time $\tau_{Z}$ as the first $t \in[0,1,2, \ldots]$ that provides minimum of the expression

$$
p_{U}(t)=\left|\left\langle\psi_{0}\left|U^{t}\right| \psi_{0}\right\rangle\right|^{2}
$$

Obviously, the expression (14) is either constant (namely, $p_{U}(t)=1$ ) or periodic. In the latter case its period is a divisor of the order of $U$. The order of an element $a$ of a group is the smallest natural number $n>0$ such that $a^{n}=e$, where $e$ denotes the identity element of the group. The order of $a$ will be denoted ord $(a)$. For the faithful representation, ord $(U) \equiv \operatorname{ord}(\mathrm{U}(\mathrm{g}))=\operatorname{ord}(\mathrm{g})$.

Consider, for example, the "Max-Zehnder" representation $\mathrm{U}_{M Z}$ of the group $\mathbb{Z}_{8}$, i.e., the "beam splitter" matrix (1) is taken as a generator of $\mathbb{Z}_{8}$. Table 1 presents the Zeno times for all operators from the representation $\mathrm{U}_{M Z}$. We adopt the convention (motivated by formula (13)) that $\tau_{Z}=\infty$ if the probability (14) is constant.

Table 1. Zeno times for all operators from $\mathrm{U}_{M Z}\left(\mathbb{Z}_{8}\right)$

\begin{tabular}{c|c|c|c}
$U=\mathrm{U}_{M Z}(\mathrm{~g})$ & $\operatorname{ord}(\mathrm{g})$ & $\operatorname{Period}\left(p_{U}(t)\right)$ & $\boldsymbol{\tau}_{Z}$ \\
\hline$S^{0}=\mathrm{I}$ & 1 & $p_{U}(t)=1$ & $\infty$ \\
\hline$S^{4}$ & 2 & $p_{U}(t)=1$ & $\infty$ \\
\hline$S^{2}=M, S^{6}$ & 4 & 2 & 1 \\
\hline$S, S^{3}, S^{5}, S^{7}$ & 8 & 4 & 2
\end{tabular}

The two-dimensional "Max-Zehnder" representation $\mathrm{U}_{M Z}$ can be generalized to the arbitrary cyclic group $\mathbb{Z}_{N}$ by replacing the "beam splitter" matrix of the form (2) with the unitary matrix

$$
S_{N}=\frac{1}{2}\left(\begin{array}{ll}
\mathrm{r}+\mathrm{r}^{N-1} & \mathrm{r}-\mathrm{r}^{N-1} \\
\mathrm{r}-\mathrm{r}^{N-1} & \mathrm{r}+\mathrm{r}^{N-1}
\end{array}\right),
$$


where $\mathrm{r}$ is an $N$ th primitive root of unity. Figure 4 shows the evolution of the probability to observe the initial state for the evolution operator $S_{100}$ in the time interval $0 \leq t \leq 100$. The quadratic shorttime behavior, described by the formula (13), is clearly visible in the figure. The Zeno time in this example is $\tau_{Z}=25$.

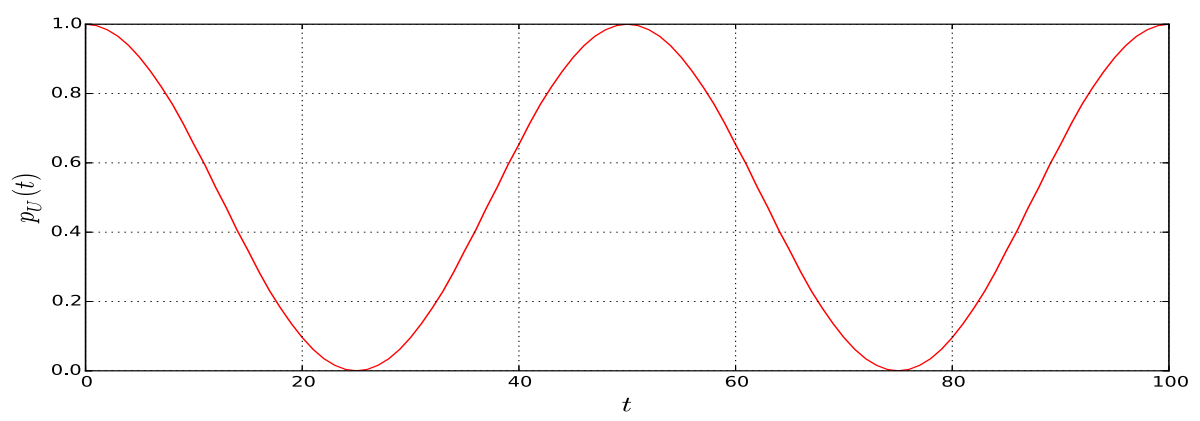

Figure 4. Probability $p_{U}(t)$ vs. time $t$ for the operator $U=S_{100} \in \mathrm{U}_{M Z}\left(\mathbb{Z}_{100}\right)$.

As a non-commutative example, consider the icosahedral group $A_{5}$ - the smallest $\left(\left|A_{5}\right|=60\right)$ non-commutative simple group. It has applications for model building in the particle physics, especially in issues beyond the standard model, such as the flavor physics [11]. The non-trivial elements of $A_{5}$ have orders 2, 3 and 5. The irreducible representations of $A_{5}$ are: one trivial singlet, $\mathbf{1}$, two triplets, $\mathbf{3}$ and $\mathbf{3}^{\prime}$, one quartet, $\mathbf{4}$, and one quintet, $\mathbf{5}$. Figure 5 shows the evolution of the "Zeno probabilities" for the following matrices of orders 2,3 and 5 , respectively,

$$
U=\frac{1}{2}\left(\begin{array}{ccc}
-\phi & 1 / \phi & 1 \\
1 / \phi & -1 & \phi \\
1 & \phi & 1 / \phi
\end{array}\right), V=\left(\begin{array}{ccc}
0 & 0 & 1 \\
1 & 0 & 0 \\
0 & 1 & 0
\end{array}\right), W=\frac{1}{2}\left(\begin{array}{ccc}
-\phi & -1 / \phi & 1 \\
1 / \phi & 1 & \phi \\
-1 & \phi & -1 / \phi
\end{array}\right)
$$

where $\phi=\frac{1+\sqrt{5}}{2}$ is the "golden ratio". To write these matrices, we added an element of order 3 (the simplest among randomly selected) to the generators of orders 2 and 5 proposed in [12] for the representation $\mathbf{3}^{\prime}$.

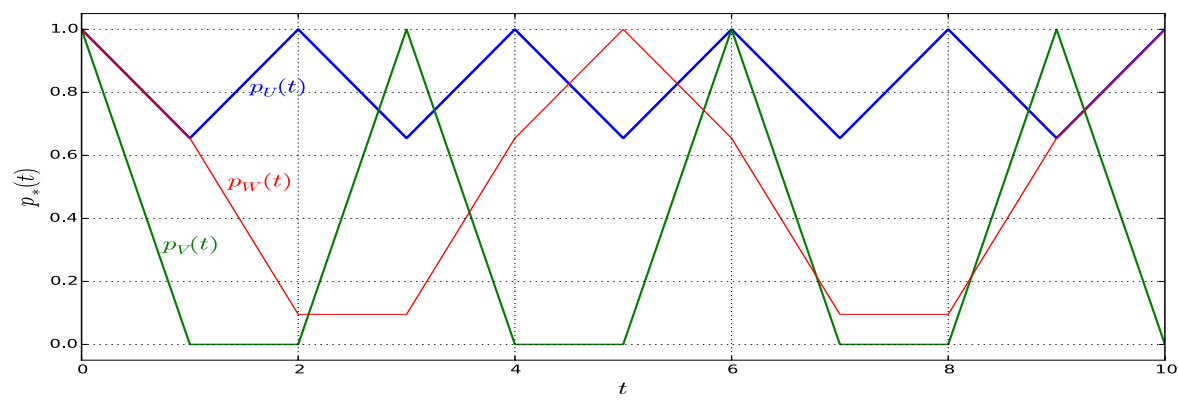

Figure 5. Zeno dynamics in the representation $\mathbf{3}^{\prime}$ of the group $A_{5}$ for unitary operators (15) 


\section{Summary}

1. We adhere to the idea of empirical universality of discrete, more specifically, finite models for describing physical reality. In other words, any continuous model can be replaced by a finite model that fit the same observable behavior.

2. This idea, in application to quantum problems, means that unitary groups of evolution operators can be replaced by unitary representations of finite groups.

3. The mathematical fact that any representation of a finite group can be embedded in a permutation representation allows to approximate, with arbitrary precision, the quantum amplitudes by projections of vectors with natural components. The complex components of these projections are combinations of natural numbers and roots of unity.

4. To illustrate the content of the article, we have used the Mach-Zehnder interferometer - a simple but important example of a two-level quantum system with rich behavior.

5. We propose a scheme for constructing quantum models. Taking into account that a single unitary evolution, being a simple change of coordinates, is not sufficient to describe the physical phenomena, the scheme involves sequences of observations with bunches of unitary parallel transports between the observations.

6. The principle of selection of the most probable trajectories in such models via the large numbers approximation leads in the continuum limit to the principle of the least action with appropriate Lagrangians and deterministic evolution equations.

7. To look at the connection between quantum dynamics and the group properties of unitary evolution operators, we have considered the quantum Zeno effect in the context of our approach.

\section{Acknowledgements}

The work is supported in part by the Ministry of Education and Science of the Russian Federation (grant 3003.2014.2) and the Russian Foundation for Basic Research (grant 13-01-00668).

\section{References}

[1] Kornyak V.V., Phys. Part. Nucl. 44, 47-91 (2013); http://arxiv.org/abs/1208.5734

[2] Magnus W., Bull. Amer. Math. Soc.75, No 2, 305-316 (1969)

[3] Kornyak V.V., J. Phys.: Conf. Ser. 343 (2012) 012059 http://iopscience.iop.org/1742-6596/343/1/012059

[4] Kornyak V.V., J. Phys.: Conf. Ser. 442 (2013) 012050 http://iopscience.iop.org/1742-6596/442/1/012050

[5] Gleason A.M., Indiana Univ. Math. J. 6, 885-893 (1957)

[6] Elitzur A. and Vaidman L., Foundation of Physics 23, 987-997 (1993)

[7] Reck M., Zeilinger A., Bernstein H.J., Bertani P., Phys. Rev. Lett. 73, 58-61 (1994)

[8] Kornyak V.V., Math. Model. Geom. 3, 1-24 (2015); http://arxiv.org/abs/1501.07356

[9] Knuth K.H., AIP Conf. Proc. 1641, 588 (2015); http://arxiv.org/abs/1411.1854

[10] Facchi P., Pascazio S., J. Phys. A: Math. Theor. 41 (2008) 493001 doi:10.1088/1751-8113/41/49/493001

[11] Everett L.L., Stuart A.J., Phys. Rev. D 79, 085005 (2009); http://arxiv.org/abs/0812.1057

[12] Shirai K., J. Phys. Soc. Jpn. 61, 2735-2747 (1992) 